\title{
Demonstration Abstract: Crowdsourced Indoor Localization and Navigation with Anyplace
}

\author{
${\text { Lambros Petrou*, George Larkou*, Christos Laoudias }{ }^{\dagger} \text {, Demetrios Zeinalipour-Yazti* and Christos G. Panayiotou }}^{\dagger}$ \\ * Department of Computer Science, University of Cyprus, 1678 Nicosia, Cyprus \\ ${ }^{\dagger}$ KIOS Research Center for Intelligent Systems and Networks, University of Cyprus, 1678 Nicosia, Cyprus
}

\begin{abstract}
In this demonstration paper, we present the Anyplace system that relies on the abundance of sensory data on smartphones (e.g., WiFi signal strength and inertial measurements) to deliver reliable indoor geolocation information. Our system features two highly desirable properties, namely crowdsourcing and scalability. Anyplace implements a set of crowdsourcing-supportive mechanisms to handle the enormous amount of crowdsensed data, filter incorrect user contributions and exploit WiFi data from heterogeneous mobile devices. Moreover, Anyplace follows a big-data architecture for efficient and scalable storage and retrieval of localization and mapping data.
\end{abstract}

Keywords-Crowdsourcing, indoor localization, navigation.

\section{INTRODUCTION}

Crowdsourcing has recently emerged as a promising solution for collecting the high volume of location-tagged data, e.g., the WiFi Received Signal Strength (RSS) radiomap of a multi-storey building, which are required to support indoor localization systems. In this context, volunteers engage in participatory sensing campaigns to collect location-dependent RSS samples from the surrounding WiFi Access Points (AP) that they later contribute to the system. This is an attractive approach, because it splits the cumbersome and time consuming data collection task among the crowd. At the same time, however, it raises new challenges such as filtering incorrect contributions (trustworthiness), managing the radiomap size and fusing data from heterogeneous mobile devices that report the RSS measurements very differently.

Several systems leverage crowdsourcing for creating and updating the RSS radiomap, including Redpin [1], our awardwinning Airplace platform [2] and others. However, these systems do not consider diverse devices. The WiFiSLAM application [3] and the Molé system [4] allow the user to carry any device during localization. Still, they require a homogeneous radiomap, i.e., collected with a single device or by contributors carrying the same devices, thus they cannot exploit the full benefit of crowdsourcing. Other systems, such as Elekspot [5] and FreeLoc [6], support different devices both for creating the radiomap and localizing, however they do not cope with the radiomap size and data quality issues.

We address the above challenges with efficient mechanisms at the server side. Regarding scalability issues, our system operates on top of a big-data management back-end service, with a Google Maps user interface. Anyplace ${ }^{1}$ will allow entities (users, companies, organizations, etc.) to realize indoor information management systems, including point of interest

\footnotetext{
${ }^{1}$ The Anyplace system, https://anyplace.cs.ucy.ac.cy/
}

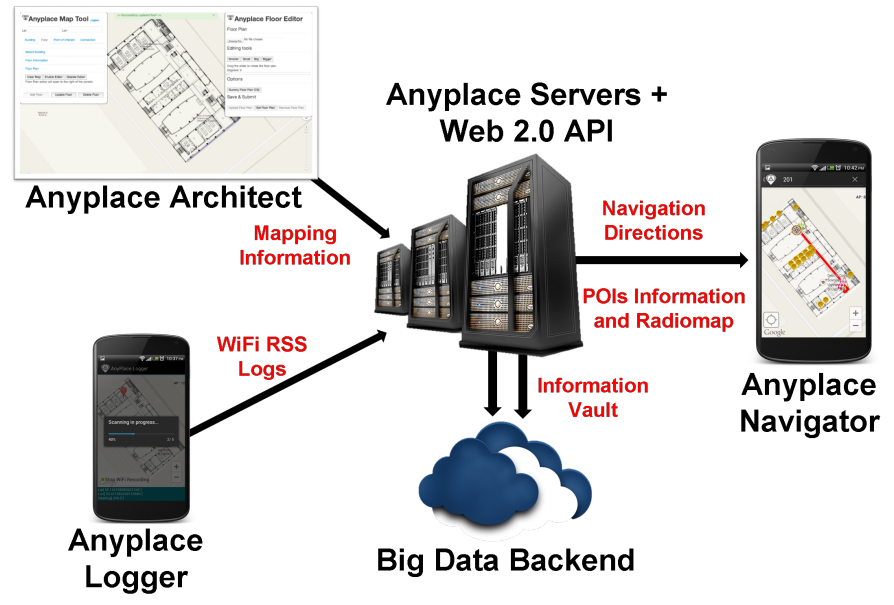

Fig. 1. The Anyplace system architecture.

(POI) navigation, on top of existing wireless networks by leveraging rich multi-sensory data available on smartphones.

\section{Anyplace System ARChitecture}

Our platform consists of four components, including the Server, the Architect and two client applications running on Android smartphones, namely the Logger and the Navigator. The Anyplace system architecture is shown in Fig. 1.

\section{A. Anyplace Server \& API}

The Server follows a big-data architecture and provides a Web 2.0 API using JSON objects for Mapping, Navigation and Localization. It stores buildings, floorplan maps, and POIs information, while it uses Couchbase as its back-end database for scalability and fast metadata retrieval. Moreover, the Server delivers indoor navigation directions to the user upon request.

In addition, the Server features several modules to assist the crowdsourcing functionality. Importantly, the differential RSS module outputs RSS differences, instead of absolute RSS values, for fusing the data contributed from diverse devices into a single usable radiomap [7]. To keep the size of the radiomap low, the spatial aggregation module spans a grid over the area with predefined resolution (e.g., $2 \times 2$ meters) and aggregates all the uploaded RSS values from the same WiFi AP that fall within the same grid cell. In addition, the outlier filtering module checks the validity of the contributed RSS data by comparing the MAC addresses of the APs at the presumed user location against the relevant data in the radiomap. 


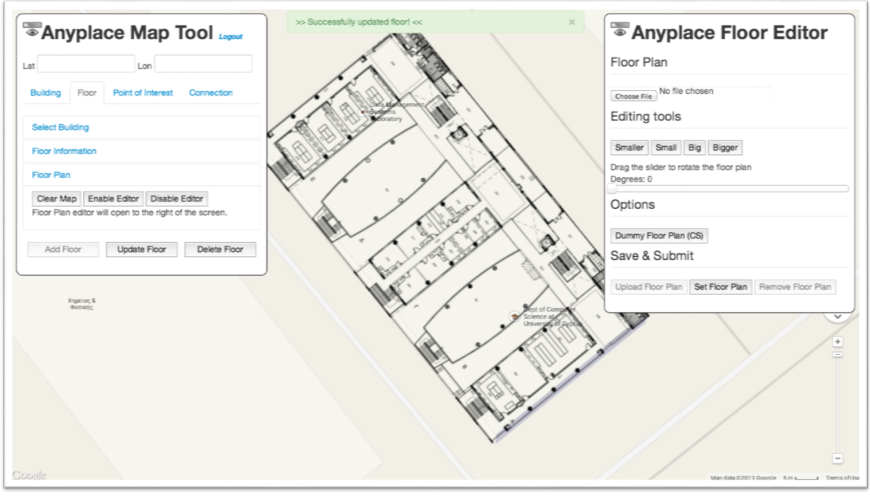

Fig. 2. The Anyplace Architect mapping tool and floor editor.

\section{B. Anyplace Architect}

The Architect website offers a feature-rich, user-friendly and account-based interface for placing the blueprint of a building on top of Google Maps with multi-floor support. In particular, within the Architect floor editor the user is able to upload, scale and rotate the desired blueprints to fit them properly, as shown in Fig. 2. The user can later add, annotate and geo-tag POIs inside the building and connect them to indicate feasible paths for enabling the delivery of navigation directions. The Architect also offers a Viewer mode to provide read-only access to building, floorplan and POI information.

\section{Anyplace Logger}

The Logger application enables users to record RSS readings from nearby WiFi APs and upload them to our Server through the API. In this fashion, volunteers are contributing RSS data for crowdsourcing the radiomap. Contributors can select the desired building and floor, as well as modify the number of samples to be recorded and other settings, through the preferences screen. Subsequently, the users indicate their current location by clicking on the map and then click the on-screen buttons to initiate and end the logging process.

\section{Anyplace Navigator}

The Navigator allows users to see their current location on top of the floorplan map and navigate between POIs inside the building. The onboard smartphone sensors (i.e., accelerometer, gyroscope and digital compass) are seamlessly integrated in our tracking module to smooth the WiFi locations and enhance the navigation experience. When the Navigator is launched, the building map and the associated POIs are automatically loaded by using the rough user location provided by the Google Geolocation API (Fig. 3, left). Then, the application downloads the RSS radiomap of the relevant floor and displays the user location on top of the map, using a WiFi localization algorithm developed in-house, while the user can click on nearby POIs to get more information (Fig. 3, middle). Moreover, users may search for POIs and get navigation directions from their current location to the desired POI (Fig. 3, right).

\section{DEMONSTRATION EXPERIENCE}

Our system has low setup time because it will rely on the WiFi infrastructure available at the conference venue. If neces-
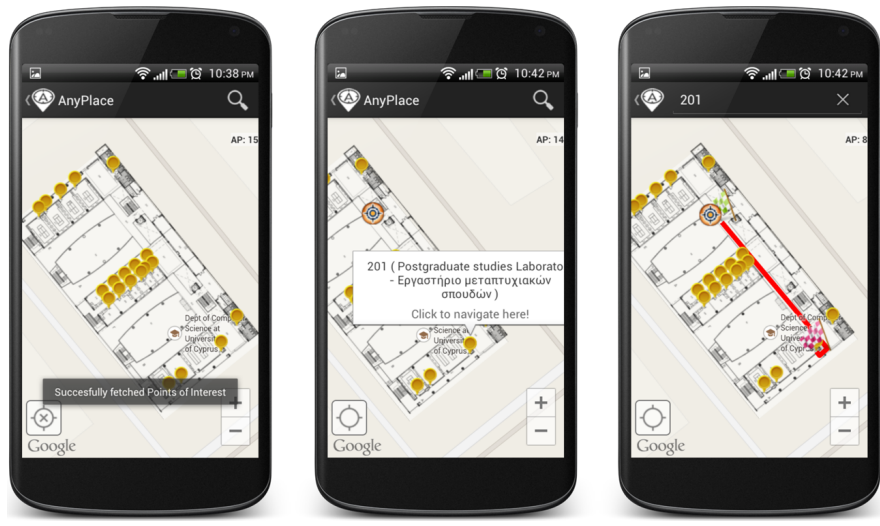

Fig. 3. Screenshots of the Anyplace Navigator application.

sary, our team can quickly deploy additional smartphone APs to provide adequate $\mathrm{WiFi}$ coverage. Thus, only the floorplan digital map of the demo site is required.

We will demonstrate the real-time localization capabilities of Anyplace by allowing attendees to carry an Android smartphone running the Navigator software and viewing their travelled path, while walking around the demo area. At the same time the participants will be able to appreciate the potential of indoor location-oriented services by carrying out POI search and navigation.

They will also have the opportunity to interact with the Architect website in order to add and annotate indoor spaces in a straightforward way or simply explore other annotated buildings that are publicly available through the Viewer mode.

\section{ACKNOWLEDGMENT}

This work has been funded by the University of Cyprus and supported by EU's COST Action IC903 (MOVE). It was co-funded by the European Regional Development Fund and the Republic of Cyprus through the Research Promotion Foundation (New Infrastructure Project/Strategic/0308/26).

\section{REFERENCES}

[1] P. Bolliger, "Redpin - adaptive, zero-configuration indoor localization through user collaboration," in 1st ACM Intl. Workshop on Mobile Entity Localization and Tracking in GPS-less environments (MELT), 2008, pp. $55-60$.

[2] C. Laoudias, G. Constantinou, M. Constantinides, S. Nicolaou, D. Zeinalipour-Yazti, and C. G. Panayiotou, "The Airplace indoor positioning platform for Android smartphones," in 13th IEEE Intl. Conference on Mobile Data Management (MDM), 2012, pp.312-315.

[3] R. Empson, "Apple acquires indoor GPS startup WiFiSlam for \$20M," Techcrunch.com, March 2013. [Online]. Available: http://goo.gl/WI3g6

[4] J. Ledlie, J.-g. Park, D. Curtis, A. Cavalcante, L. Camara, A. Costa, and R. Vieira, "Molé: a scalable, user-generated WiFi positioning engine," Journal of LBS, Taylor \& Francis, Inc, vol. 6, no. 2, pp. 55-80, 2012.

[5] M. Lee, S. H. Jung, S. Lee, and D. Han, "Elekspot: a platform for urban place recognition via crowdsourcing," in 12th IEEE/IPSJ Intl. Symposium on Applications and the Internet (SAINT), 2012, pp. 190-195.

[6] S. Yang, P. Dessai, M. Verma, and M. Gerla, "FreeLoc: calibration-free crowdsourced indoor localization," in 32nd IEEE Intl. Conference on Computer Communications INFOCOM, 2013.

[7] C. Laoudias, D. Zeinalipour-Yazti, and C. G. Panayiotou, "Crowdsourced indoor localization for diverse devices through radiomap fusion," in Intl. Conference on Indoor Positioning and Indoor Navigation (IPIN), 2013. 